\title{
What to Expect When You're Expecting: Femtoscopy at the LHC
}

\author{
Mike Lisa ${ }^{1}$ \\ ${ }^{1}$ Department of Physics, Ohio State University, \\ 1040 Physics Research Building, \\ 191 West Woodruff Ave., Columbus, OH 43210, USA
}

Received on 20 January, 2007

\begin{abstract}
A huge systematics of femtoscopic measurements have been used over the past 20 years to characterize the system created in heavy ion collisions. These measurements cover two orders of magnitude in energy, and with LHC beams imminent, this range will be extended by more than another order of magnitude. Here, I discuss theoretical expectations of femtoscopy of $A+A$ and $p+p$ collisions at the LHC, based on Boltzmann and hydrodynamic calculations, as well as on naive extrapolation of existing systematics.
\end{abstract}

Keywords: LHC; HBT; Femtoscopy; Predictions; Hydrodynamics; Boltzmann cascade; Heavy ions; RHIC

\section{INTRODUCTION}

What distinguishes ultrarelativistic heavy ion physics from particle physics is its focus on geometrically large systems. The desire is not to understand fundamental processes, as in the latter field, but to create and probe new states of matter and access the most crucial and characteristic feature of QCD- the nature of deconfinement. The geometrically-sensitive, bulk properties are the crucial ones, and these are reflected in the soft $\left(p_{T} \sim \Lambda_{Q C D}\right)$ sector.

In soft sector observables, long-term baselines have been established over a large energy range. Prior to first data at RHIC, it was commonly speculated (and hoped) that large deviations from these systematics (e.g. $\pi^{0} / \pi^{ \pm}$ratios, sidewards flow, strangeness enhancement, total multiplicity) would signal clearly the qualitatively different nature of the system created there [1]. In femtoscopic systems, rather generic arguments led to expectations $[2,3]$ of a rapid increase, with $\sqrt{s_{N N}}$, in the pion "HBT radii" $R_{\text {out }}$ and $R_{\text {long }}$, reflecting relatively long timescales of the transition from deconfined QGP to confined hadronic matter.

Such dramatic speculations are largely absent today, in anticipation of LHC collisions. Soft-sector, global observables at RHIC are only quantitatively different than they are at lower energies. Even in the high- $p_{T}$ sector, where jet suppression and partonic energy loss measurements have generated huge excitement, energy scans at RHIC reveal that the data indicate more of an evolution than a revolution.

This is all to the good. Discoveries via sharp jumps à la superconductivity are not our lot. The real science behind heavy ion measurements (at very high energies as well as at much lower ones) lies in understanding the details of the data.

Femtoscopy [4], the geometric measurement of systems at the fermi scale, has been used to characterize the freezeout substructure of heavy ion collisions for two decades in time, and over two decades (orders of magnitude) in collision energy [5]. Soon, this energy range will be extended by another decade at the LHC.

It will be important to understand the evolution of the nontrivial space-time substructure of the bulk system as the initial conditions change dramatically with energy. In Fig. 1 is shown the number of refereed-journal papers of experimental femtoscopic results in relativistic heavy ion collisions, as a

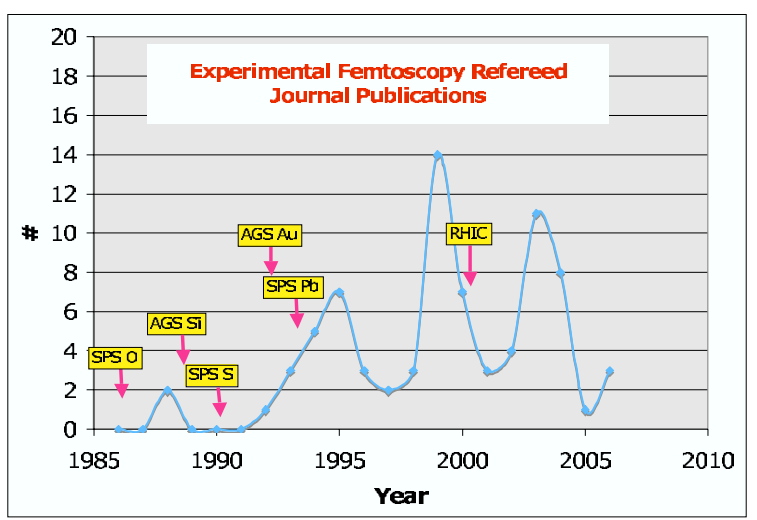

FIG. 1: (Color online) The number of refereed-journal publications reporting new femtoscopy results in relativistic heavy ion collisions. Beginning of availability of new beams are indicated in yellow boxes.

function of publication year. Even armed with nothing more than this Figure and Reinhard Stock's observation [6] that "HBT experiences a renaissance of new insights roughly every five years," we may confidently expect a barrage of new femtoscopic information to digest within the next 5 years.

Here, we discuss predictions for femtoscopy at the LHC. In the next Section, we consider the case of simple extrapolation of measured femtoscopic trends, with no reference to physics per se. In Section III, we consider predictions of Boltzmann/cascade transport calculations, and in Section IV those of hydrodynamical models. Finally, we discuss speculations on the physics behind femtoscopic measurements in $p+p$ collisions, which will, in fact, be the first results available at the LHC. At the end we summarize.

\section{NOTHING NEW UNDER THE SUN (NNUS) SCENARIO}

Femtoscopic measurements display rich, multidimensional and nontrivial systematic dependences upon kinematic $\left(p_{T}\right.$, $y$, etc) variables and particle species [5, 7]. The dependence upon global variables such as $\sqrt{s_{N N}}$ and impact parameter, however, appears significantly more trivial. Schematically characterizing the measured femtoscopic length scales as a multidimensional function, evidence thus far indicates an 


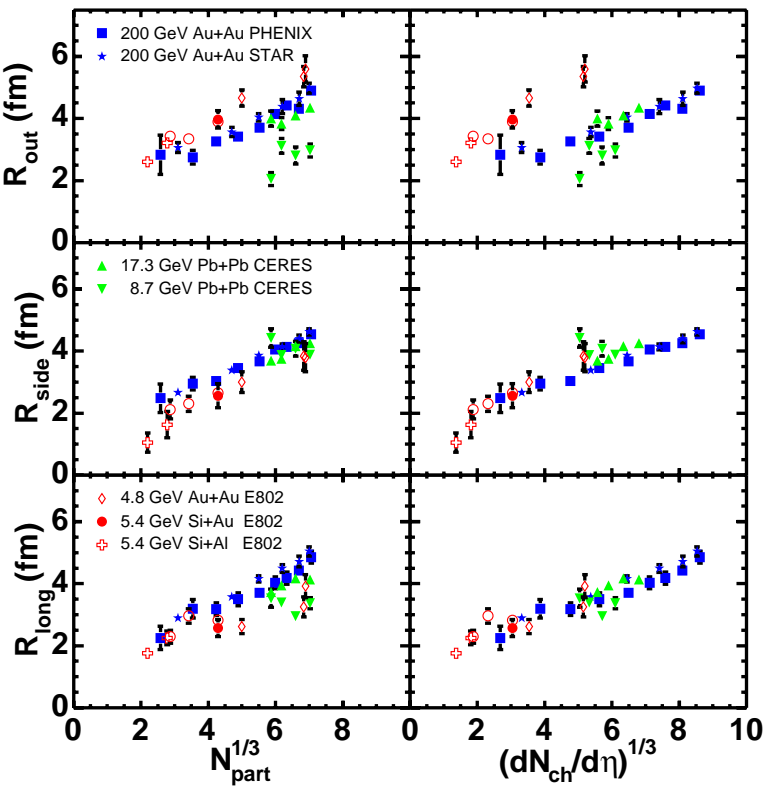

FIG. 2: (Color online) Pion HBT radii plotted versus the number of participating nucleons (left) and versus the charged particle multiplicity (right) for collisions of varying centrality and a wide range of energies. Compilation from [5].

overall factorization

$$
\begin{array}{r}
R\left(\sqrt{s_{N N}}, A, B,|\vec{b}|, \phi, y, m_{T}, m_{1}, m_{2}\right) \\
=R_{g}\left(\sqrt{s_{N N}}, A, B,|\vec{b}|\right) \cdot F_{k}\left(\phi, y, m_{T}, m_{1}, m_{2}\right) \\
=R_{g}(M) \cdot F_{k}\left(\phi, y, m_{T}, m_{1}, m_{2}\right),
\end{array}
$$

where $F_{k}$ is a dimensionless function containing, e.g. decreasing "HBT radii" with particle $m_{T}$. The dimensional scale $R_{g}$ is determined by global observables. However, as indicated by the second equality of Equation 1, to good approximation the only relevant global observable is the total multiplicity $M$ of the collision. In fact, this multiplicity dominance well apply to all soft-sector observables [8].

There are at least three caveats to the above statement. Firstly, the CERES [9] collaboration has shown that the scale depends also on the freeze-out chemistry (baryon-to-meson ratio) in addition to the multiplicity. This is important at low (AGS) energies. For collisions above top SPS energy, $\sqrt{s_{N N}} \sim 17 \mathrm{GeV}$, the chemical evolution is sufficiently weak that one may consider multiplicity only. Secondly, as seen in Fig. 2, there is some residual dependence of the outward radius on $\sqrt{s_{N N}}$ in addition to multiplicity; in this Section, we ignore this potentially important detail. Finally, the azimuthal $\left(\phi_{p}\right.$, as determined relative to the reaction plane) dependence [10-13] likely at some point violates the factorization. While it has not been experimentally tested, two collisions producing the same multiplicity, one very peripheral (i.e. spatially anisotropic in the entrance channel) at high energy and the other very central at low energy, presumably generate freezeout distributions with different spatial anisotropy, which is then reflected in the azimuthally-sensitive femtoscopy [14]. See Section IV for further discussion.

These caveats stated, however, the factorization of Equation 1 is probably our best, zero-new-physics guide to simple extrapolation of femtoscopic trends measured over two orders of magnitude in $\sqrt{s_{N N}}$ and from from the lightest $(p+p)$ to the heaviest $(P b+P b)$ systems. Fig. 2 suggests a simple form $R_{g}(M) \propto M^{1 / 3}$; this ignores the finite offset $\sim 1 \mathrm{fm}$ when extrapolating $M \rightarrow 0$, but this is negligible for high multiplicity. This relation may reflect that a constant freezeout density drives the femtoscopic scales [9], though this neglects any dynamic effects. Assuming that this simple proportionality continues, then, we know $R_{g}(M)$ and determining femtoscopic expectations boils down to anticipating the multiplicity at the LHC.

A naive extrapolation [7, 8] of systematics suggests that $d N / d y$ at the LHC will be $60 \%$ larger than that observed at RHIC. Thus, the zeroth-order expectation is that length scales at the LHC will be $17 \%\left(1.6^{1 / 3}=1.17\right)$ larger than those measured at RHIC, for all kinematic selections and particle species, according to Equation 1.

Going beyond simple extrapolation to include a physical picture, saturation-based calculations [15] give much higher multiplicity- roughly triple that at RHIC. This leads to expectations of length scales $45 \%$ higher than those at RHIC. Thus, $R_{\text {long }}$ for pions at midrapidity and low $p_{T}$ in central collisions would be $1.45 \times 7 \mathrm{fm}=10 \mathrm{fm}$.

Multiplicity predictions based on Boltzmann/cascade calculations can be significantly higher yet. Selecting two for which femtoscopic predictions also exist (Section III), A Multi-Phase Transport (AMPT) calculation [16] and the Hadronic Rescattering Model (HRM) [17] predict $5 \times$ and $7 \times$ RHIC multiplicity, respectively. Thus, femtoscopic scales at LHC may be as much as $90 \%$ higher than at RHIC. Depending on the final-state interaction which produces the two-particle correlation function, measuring length scales of $\sim 15 \mathrm{fm}$ may challenge experimental two-track resolutions. For two-pion correlations, such scales are within the capabilities of the ALICE detector [18].

\section{BOLTZMANN TRANSPORT CALCULATIONS}

More interesting than simple scaling relations are models with real physics and dynamics, such as transport calculations. Boltzmann/cascade transport models generally reproduce "HBT radii" at RHIC better than do hydrodynamic calculations [5]. The reasons behind this include different physics in the models, a more detailed description of the kinetic freezeout, and the use of more appropriate methods of calculating the radii [19]. Predictions of pion HBT radii with each of the transport calculations discussed in Section II reveal predictions more subtle than the simple multiplicityscaling discussed above.

For an infinite and boost-invariant system (only an approximation of reality, of course), the longitudinal HBT radius $R_{\text {long }}$ is proportional to the system evolution time (i.e. between interpenetration of the ions and kinematic freezeout of the products) $[5,20]$. Naturally, this is not a unique, 


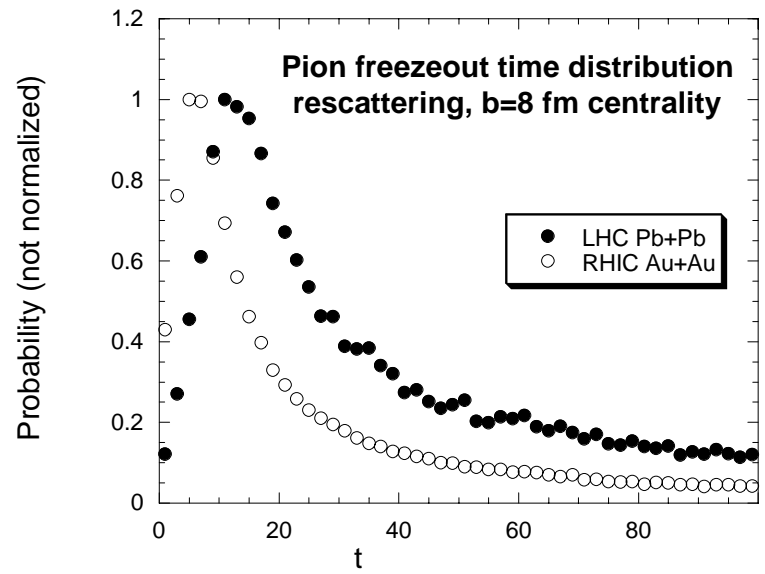

FIG. 3: The freezeout time distribution for midrapidity pions from the Hadronic Rescattering Model of Humanic [17] for RHIC and LHC conditions.

system-wide time, but a distribution. An example is seen in Fig. 3, in which the freeze-out time distribution for midrapidity pions from collisions at RHIC and LHC are compared in the HRM calculation. The LHC timescales are roughly double those at RHIC. Although HRM is not explicitly a boost-invariant model, we see in Fig. 4 that $R_{\text {long }}$ reflects this timescale increase, roughly doubling when the energy is increased from RHIC to LHC energies. The $\sim 70 \%$ increase in $R_{\text {long }}$ is roughly consistent, then, with expectations from both a timescale and from the multiplicity-scaling point of view. This is certainly not a coincidence, as the increased timescale is due in large part to the increased multiplicity.

On the other hand, there is more going on. The predicted $p_{T}$-dependence of both $R_{\text {long }}$ and $R_{\text {side }}$ are steeper at the LHC than at RHIC. Also, the increase in $R_{\text {side }}$ is significantly less than $90 \%$. Both of these effects are consistent with a freezeout scenario with significantly increased transverse flow [14]. Indeed, transverse momentum distributions predicted by HRM are significantly harder (less steep) at the LHC than those at RHIC. Since the $p_{T}$ dependence of HBT radii [5] and spectra in the soft sector are observed to change very little between $\sqrt{s_{N N}}=20 \div 200 \mathrm{GeV}$, it will be interesting to see whether this trend is broken at the LHC, as predicted by HRM.

The HRM model is a deliberate effort to use the simplest (often criticized as too simplistic) physics picture, free of novel phases like QGP. It is a pure hadron-based transport calculation, though the initial conditions may be taken from Pythia or Saturation-based scenarios [17]. On the other side of the "simplicity spectrum" is AMPT, an attempt to describe the various stages of the system's evolution in terms of the most appropriate model for that stage [21].

Similar to HRM, AMPT predicts stronger transverse flow at the LHC, as compared to RHIC, leading to steeper $p_{T^{-}}$ dependence of HBT radii. In terms of scale, the transverse (longitudinal) radii are predicted to increase by $10 \%(30 \%)$. This is more modest than the predictions of HRM $(30 \%$ and $70 \%$, respectively), and much more modest than puremultiplicity scalings of Section II.

Thus the dynamical physics, in these models, lead to ex-

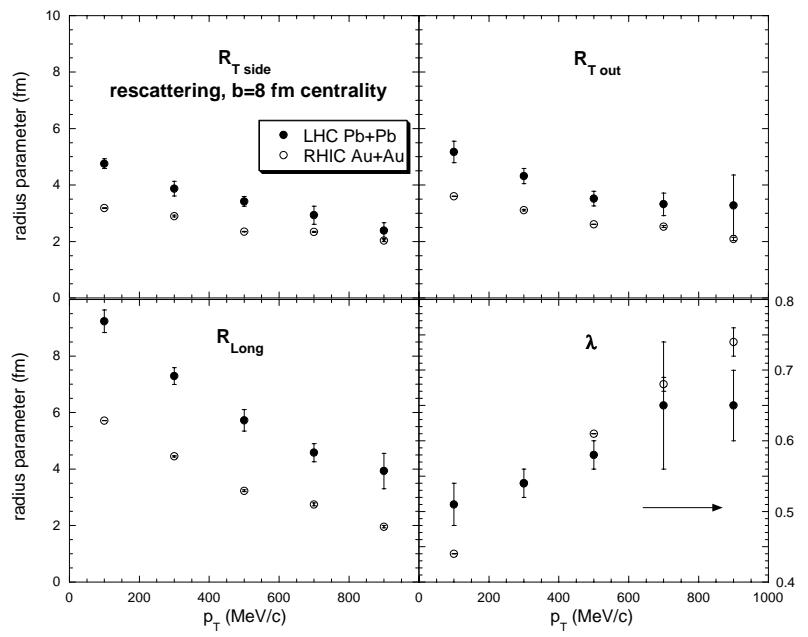

FIG. 4: HBT radii from fits to pion correlation functions from the Hadronic Rescattering Model of Humanic [17] for RHIC and LHC conditions.

pected details significantly beyond simple extrapolation of lower-energy results.

\section{HYDRODYNAMICAL CALCULATIONS}

As mentioned, hydrodynamical models tend to reproduce femtoscopic measurements more poorly than do Boltzmann/cascade calculations. On the other hand, they have enjoyed huge success in reproducing momentum-space observables such as elliptic flow. Furthermore, the conditions at LHC are likely to provide an even better approximation than at RHIC to the zero-mean-free-path assumptions of pure hydrodynamics. Finally, the direct connection between hydrodynamics and the Equation of State of strongly-interacting matter (color-confined or not) remains a compelling reason to explore soft-sector, bulk consequences of the model.

\section{A. Source Length Scales}

Recently, Eskola and collaborators [22] coupled a pQCD+saturation-based prediction for initial conditions at LHC to their 1+1-dimensional hydro calculation. The Equation-of-State featured a first-order phase transition between an ideal QGP at high temperature and a hadron resonance gas at low temperature.

As shown in Fig. 5, the initial energy density at which hydrodynamics is assumed to take over expected to roughly an order of magnitude larger at the LHC than at RHIC, due both to increased gluon production and to shorter system formation (thermalization) time $\tau_{0}$ at the higher energy. Since the initial transverse scale changes only little, the pressure gradients will likewise be much higher at LHC, leading to increased transverse flow. These effects place competing pressures on the space-time evolution of the system, and on the femtoscopic scales at freezeout, as discussed below.

The increased energy density (directly associated with entropy density and thus multiplicity) tends to produce longer timescales at the LHC. Longitudinal expansion tends to cool 


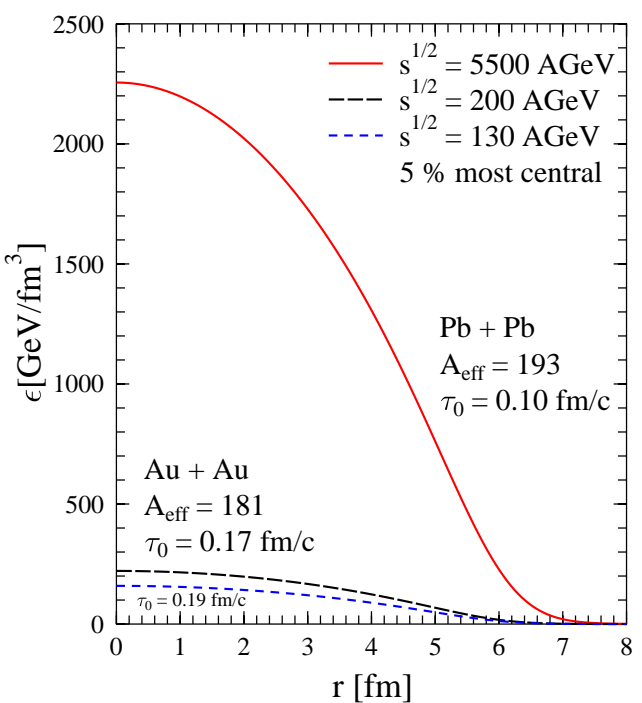

FIG. 5: (Color online) Initial energy density distribution in the transverse plane, calculated by Eskola et al [22].

the system towards freezeout conditions. However, especially at the LHC, the large transverse flow generated by the intense pressure gradients cannot be ignored. Eskola and collaborators [22], estimate that the time required to cool from the maximum energy density (at $r=0$ in Fig. 5 to the critical energy density $\left(\varepsilon_{c}=1.93 \mathrm{GeV} / \mathrm{fm}^{3}\right)$ would be $6 \mathrm{fm} / \mathrm{c}(20 \mathrm{fm} / \mathrm{c})$ at RHIC (LHC), due to longitudinal expansion alone. However, when transverse dynamics are included, the cooling times become $5 \mathrm{fm} / \mathrm{c}(7.5 \mathrm{fm} / \mathrm{c})$ at RHIC (LHC). The evolution time to kinematic freezeout- say until $T \approx 140 \mathrm{MeV}-$ is $\tau_{0} \sim 12-14 \mathrm{fm} / \mathrm{c}$ in both cases; this is the timescale most directly probed by femtoscopy. This is dramatic- the effect of transverse flow on cooling timescales can almost be neglected at RHIC, while it is dominant at the LHC. This is reminiscent of the cascade calculations discussed in Section III; the much stronger flow may well lead to deviations from the trends (e.g. $p_{T}$-dependence of pion HBT radii being independent of $\left.\sqrt{s_{N N}}\right)$ established so far at lower energy. This aspect of the NNUS scenario may finally be violated. The qualitative difference is apparent from the freezeout hypersurfaces at RHIC and LHC, shown in Fig. 6. The Figure is from a calculation by Kolb and Heinz [23], but is similar to Eskola's.

It would be very interesting to know whether the other aspect of NNUS, namely the multiplicity scaling shown in Fig. 2, is satisfied by the hydro models. Unfortunately, Eskola did not calculate pion "HBT radii," and Heinz and Kolb did not calculate multiplicity, so a consistent estimate of the scaling cannot be checked. However, the former predict that the multiplicity at LHC will be approximately triple that at RHIC, corresponding to a $40 \%$ increase in HBT radius under NNUS scaling. Heinz and Kolb [23] do, in fact, predict roughly this increase in the transverse radii. On the other hand, the cause behind their prediction of a decrease in $R_{\text {long }}$ at LHC relative to RHIC is unclear; work is underway to better understand this effect [35].
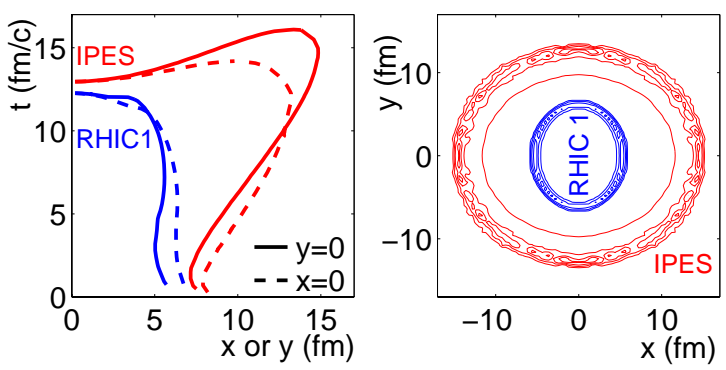

FIG. 6: (Color online) Left: freeze-out space-time hypersurface in collisions with finite impact parameter calculated by Heinz and Kolb [23]. The "IPES" calculation is an estimate of the system created at the LHC. The impact parameter is defined to lie in the $\hat{x}$-direction. Right: time-,z- and momentum-integrated freeze-out shapes in the transverse plane.

\section{B. Source Shape}

Azimuthally-sensitive pion interferometry- the measurement of spatial scales as a function of emission angle relative to the reaction plane- probes the shape of the freezeout configuration, in addition to its scale [10, 14, 24]. At finite impact parameter, both the spatial configuration of the entrance channel and the resulting momentum distribution in the exit channel are anisotropic. In particular, the initial state is spatially extended out of the reaction plane, and the resulting flow is stronger in the reaction plane (elliptic flow $v_{2}>0$ ).

Due to the preferential in-plane expansion, as the system evolves the spatial configuration should become increasingly in-plane extended (equivalently, decreasingly out-of-plane extended). Thus, knowledge of the entrance-channel shape (e.g. though Glauber model calculations) and measurement of the exit-channel shape (through femtoscopy) provide "boundary conditions" on the dynamical spacetime evolution of the anisotropic system, and probe the evolution timescale. The extracted timescale is model-dependent, requiring in principle a detailed time evolution of the flow. However, a simple estimate [25] of the timescale extracted through shape measurements and that extracted from blast-wave fits $[14,26]$ to azimuthally-integrated HBT radii are roughly consistent.

Measurements of the freezeout shape at the AGS [11] and RHIC [12, 13] indicate an out-of-plane-extended configuration. Consistent with the fact that preferential in-plane expansion (i.e. elliptic flow) is stronger at RHIC, the configuration at the higher bombarding energy is rounder. This is shown in Fig. 7, in which the transverse anisotropy is characterized by $\varepsilon \equiv\left(R_{y}^{2}-R_{x}^{2}\right) /\left(R_{y}^{2}+R_{x}^{2}\right)(x$ is in the reaction plane).

The anisotropic shapes and corresponding azimuthallyselected HBT radii have been calculated in two dynamical models. At the AGS, the transport code RQMD [27] reproduces the overall scale [28] and the anisotropy [10,11] of the source reasonably well, as shown in Fig. 7. At RHIC, the $2+1$ hydro code [23] reproduces the shape quite well, while missing the scale. At the LHC, the latter calculation predicts- 


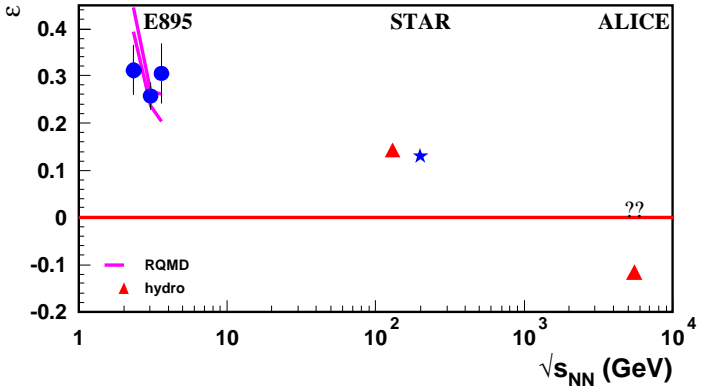

FIG. 7: (Color online) Transverse spatial freezeout anisotropy $\varepsilon$ as a function of collision energy, as estimated with azimuthally-sensitive pion femtoscopy, for collisions with impact parameter $\sim 7 \mathrm{fm}$. Round sources correspond to $\varepsilon=0 ; \varepsilon>0$ indicates an out-of-planeextended source. Measurements at the AGS [11] and RHIC [13] are compared with RQMD and hydrodynamic calculations, respectively, and the shape predicted by hydro at the LHC is shown.

again- a qualitative change in the freezeout distribution. As shown in the right panel of Fig. 6 the source is expected to evolve to an in-plane configuration $(\varepsilon<0)$. A further qualitatively new feature is the predicted sign inversion of the oscillations of the HBT radii with $p_{T}[14,23]$.

\section{PROTON COLLISIONS}

Before heavy ions are accelerated at the LHC, proton collisions at $\sqrt{s}=1.4 \mathrm{TeV}$ will be measured. While the thrust of the $p+p$ program is towards Higgs physics, it is wellrecognized that $p+p$ collisions serve as a valuable reference to heavy ion analyses in the "hard" (high- $p_{T}$ ) sector, where one looks for the effects of the medium on particles coming from well-calibrated fundamental processes.

Soft-sector analyses, too, should be performed for systems from the smallest to the largest, and the results compared. Since such analyses are assumed to measure the bulk properties, one might well hope for qualitative differences when comparing results for $p+p$ to $P b+P b$ collisions.

While pion HBT measurements have been common in both the high-energy and heavy-ion communities for many years, a direct "apples-to-apples" comparison between results from $A+A$ and $p+p$ collisions has not been possible until very recently. The STAR Collaboration at RHIC has reported the first direct comparison of pion $\mathrm{HBT}$ radii in $\mathrm{Au}+\mathrm{Au}, \mathrm{Cu}+\mathrm{Cu}$, $\mathrm{d}+\mathrm{Au}$, and $\mathrm{p}+\mathrm{p}$ collisions, using the same detector, same energy, identical techniques (event mixing, etc) to create the correlation function, identical coordinate systems and identical fitting techniques [29]. Remarkably, Gaussian fits to the correlation functions return "HBT radii" which factorize according to Equation 1 ; i.e. $F_{k}$, which quantifies the dynamically-generated substructure, is identical in $p+p$ and $A+A$ collisions. However, the STAR data show significant non-femtoscopic structures [29], which must be properly accounted for [30] before drawing firm conclusions. If the factorization is unchanged after a more sophisticated treatment, the physics implications might be dramatic. We do not discuss this here, but simply observe that the NNUS scenario is

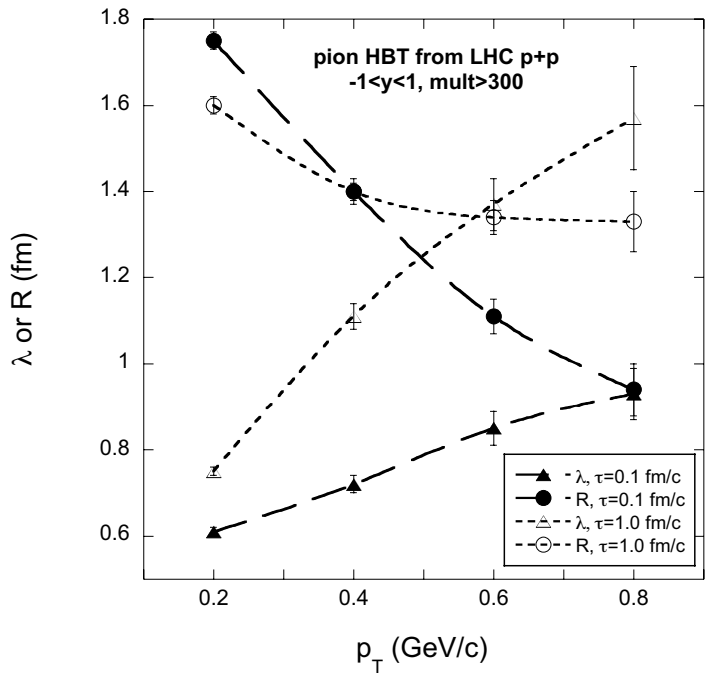

FIG. 8: Full markers: predicted $p_{T}$-dependence of $R_{i n v}$ and $\lambda$ from Gaussian fits to calculated correlation functions in a Pythia+hadronic rescattering model [31]; hadronization time was $\tau=0.1 \mathrm{fm} / \mathrm{c}$, the value required to reproduce E735 [32] data at the Tevatron. Also shown in open markers are the femtoscopic parameters corresponding to a larger $\tau$.

a likely baseline expectation for $p+p$ at LHC.

The increase of HBT radii with multiplicity has also been observed previously in $p-\bar{p}$ collisions by the E735 Collaboration [32]. While in $A+A$ collisions, this is naturally related to increasing length scales in the entrance channel geometry, Paić and Skowronski [33] postulate that jet dynamics, rather than bulk properties, drive this dependence in the $p+\bar{p}$ system. Within a simple model of hadronization, they can reproduce the E735 multiplicity dependence, and make predictions of similar multiplicity dependence for $p+p$ collisions at the top LHC energy. However, since the expectation of increasing length scales with multiplicity seems to be rather generic to all scenarios, it will be interesting to see these predictions expanded to more differential measures- say, the multiplicity and $p_{T}$ dependence, probing both aspects of Equation 1 . This should allow a more discriminating comparison between models, allowing some to be ruled out.

Such differential predictions have very recently been performed by Humanic [31], in the context of a Pythia+hadronic rescattering (through HRM) scenario. It is found that, contrary to some expectations, hadronic rescattering is crucial to understand the $M \otimes p_{T}$ dependence of the E735 data. Reproducing the data requires the assumption of a surprisingly short hadronization timescale $(\sim 0.1 \mathrm{fm})$; longer timescales do not allow sufficient hadronic rescattering needed to describe the $p_{T}$-dependence. The prediction of the model for the highest multiplicity $p+p$ collisions at $\sqrt{s}=1.4 \mathrm{TeV}$ are shown in Fig. 8 .

\section{SUMMARY}

Two decades' worth of femtoscopic systematics [5] in heavy ion collisions reveals a strikingly consistent and simple 
structure. The kinematic and particle-species dependences, which reflect dynamic substructure, decouple from the global scale, which depends (almost) solely on multiplicity. The assumption that the factorization of Equation 1 persists-i.e. that $F_{k}$ remains unchanged at the LHC- together with the assumption $R_{g}(M) \sim \sqrt[3]{M}$, is the essence of the NNUS scenario.

In the NNUS picture, all femtoscopic length scales measured at RHIC will be reproduced at LHC, only scaled up by $20-90 \%$, depending on the multiplicity prediction. Thus, the "pion HBT radius" $R_{\text {long }}$ at low $p_{T}$ might be expected in the range $(1.2 \div 1.9) \times(7 \mathrm{fm})=8.4 \div 13 \mathrm{fm}$, while the average shift between pions and kaons (about $6 \mathrm{fm}$ at RHIC [34]) would be in the range $7.2 \div 11.5 \mathrm{fm}$. However, dynamical models generally predict interesting violations of NNUS at the LHC. The significant dispersion between predictions holds out the possibility that the data will eliminate some models.

In the HRM and AMPT Boltzmann/cascade calculations, increased rescattering due to the higher density at the LHC generates much stronger global space-momentum correlations. This leads to flatter $p_{T}$ spectra for high mass particles, and to a steeper $p_{T}$-dependence of the femtoscopic length scales; that is, $F_{k}$ would pick up a $\sqrt{s}$ dependence, violating NNUS factorization. Transverse (longitudinal) scales are expected to increase $10-30 \%$ (30-70\%), relative to RHIC values.

In hydrodyanamical calculations, much higher energy densities and pressure gradients at the LHC may generate qualitatively new femtoscopic signals. Contrary to the situation at RHIC, the transversely explosive nature of the source at the LHC severely shortens the time until freezeout. The freezeout hypersurface is of a qualitatively different shape in transverse position and time; while transverse radii may increase by $40 \%$ relative to RHIC values, the longitudinal ones should expand little, and may even decrease.

The evolution timescale may also be probed by measuring the anisotropic shape of the source in coordinate space, for non-central collisions. Here again, a qualitative difference is predicted by hydrodynamics between RHIC and LHC collisions. In particular, the greatly increased flow and somewhat increased evolution time lead to predictions of an in-plane extended source, producing $\mathrm{HBT}$ radius oscillations $180^{\circ}$ out of phase with those seen at lower energy.

Probably as important as soft-physics analyses in heavy ion systems are parallel ones for $p+p$ collisions. First preliminary "apples-to-apples" comparisons of Gaussian HBT radius measurements at RHIC suggest that NNUS factorization continues to hold even for these smallest systems; it remains to be seen whether this conclusion survives more sophisticated treatment of non-femtoscopic correlations in the data, presently underway. In the context of two simple models, pion HBT radii at the LHC depend strongly on the hadronization scenario. Both predict an increase in femtoscopic freezeout scales with increasing multiplicity, which in itself will not distinguish these models from any other. However, in one, the $p_{T}$ dependence is found to depend strongly on the hadronization time and degree of subsequent hadronic scattering. Such scattering is usually ignored in treatments of $p+p$ collisions; indeed, the lack of significant rescattering is believed to be their primary virtue as a reference measurement. As hinted at by the first RHIC measurements, maybe they are not so different from $A+A$ collisions after all. More detailed measurements at the LHC may, in fact, spur a re-evaluation of ideas of the spacetime evolution of both heavy ion and hadronic collisions.

In any case, we may confidently expect considerable activity and excitement as the next mountain forms on Fig. 1.
[1] J.W. Harris and B. Muller, Ann. Rev. Nucl. Part. Sci., 46, 71 (1996).

[2] D.H. Rischke and M. Gyulassy, Nucl. Phys. A 608, 479 (1996).

[3] S. A. Bass et al., Nucl. Phys. A 661, 205 (1999).

[4] R. Lednicky and V. L. Lyuboshits, Proc. Nantes 1990 Particle correlations and interferometry in nuclear collisions, 42 (1990).

[5] M.A. Lisa, S. Pratt, R. Soltz, and U. Wiedemann, Ann. Rev. Nucl. Part. Sci. 55, 357 (2005).

[6] Reinhard Stock, J. Phys. G 30, S633 (2004).

[7] Mike Lisa, AIP Conf. Proc. 828, 226 (2006).

[8] Helen Caines, nucl-ex/0609004.

[9] D. Adamova et al., Phys. Rev. Lett. 90, 022301 (2003).

[10] M.A. Lisa, U.W. Heinz, and Urs A. Wiedemann, Phys. Lett. B 489, 287 (2000).

[11] M. A. Lisa et al., Phys. Lett. 496, 1 (2000).

[12] Randall Wells, Ph.D. thesis, Ohio State University (2002).

[13] J. Adams et al., Phys. Rev. Lett. 93, 012301 (2004).

[14] F. Retiere and M.A. Lisa, Phys. Rev. C 70, 044907 (2004).

[15] D. Kharzeev, E. Levin, and M. Nardi, Nucl. Phys. A 747, 609 (2005).

[16] Che-Ming Ko, talk at WPCF 2006, São Paulo, Brazil (2006).

[17] Thomas J. Humanic, Int. J. Mod. Phys. E 15, 197 (2006).

[18] B. Alessandro et al., J. Phys. G 32, 1295 (2006).
[19] E. Frodermann, U. Heinz, and M.A. Lisa, Phys. Rev. C 73, 044908 (2006).

[20] S. V. Akkelin and Yu. M. Sinyukov, Phys. Lett. B 356, 525 (1995).

[21] Zi-wei Lin, C. M. Ko, and Subrata Pal, Phys. Rev. Lett. 89, 152301 (2002).

[22] K. J. Eskola, H. Honkanen, H. Niemi, P. V. Ruuskanen, and S. S. Rasanen, Phys. Rev. C 72, 044904 (2005).

[23] U.W. Heinz and P.F. Kolb, Phys. Lett. B 542, 216 (2002).

[24] S. A. Voloshin and W. E. Cleland, Phys. Rev. C 54, 3212 (1996).

[25] Michael Annan Lisa, Acta Phys. Polon. B 35, 37 (2004).

[26] J. Adams et al., Phys. Rev. C 71:044906, 2005.

[27] H. Sorge, Phys. Rev. C 52, 3291 (1995).

[28] M. A. Lisa et al., Phys. Rev. Lett. 84, 2798 (2000).

[29] Z. Chajecki, Nucl. Phys. A 774, 599 (2006).

[30] Zbigniew Chajecki and Mike Lisa, nucl-th/0612080.

[31] T. J. Humanic, nucl-th/0612098.

[32] T. Alexopoulos et al., Phys. Rev. D 48, 1931 (1993).

[33] G. Paic and P. Skowronski, J. Phys. G 31, 1045 (2005).

[34] J. Adams et al., Phys. Rev. Lett. 91, 262302 (2003).

[35] U. Heinz, private comm. 